\title{
Rapid Palatal Expanders: A Review of Their Impact on Speech Sound Production
}

\author{
Ali S Alrumikan ${ }^{1}$, Aref M Althobity ${ }^{2}$, Yazeed K Alfraih ${ }^{2}$ and Mohammed S Aldossary ${ }^{2,3^{*}}$ (iD \\ ${ }^{1}$ Consultant in Orthodontics, General Directorate of Dentistry, Ministry of Health, Saudi Arabia \\ ${ }^{2}$ General Dentist, General Directorate of Dentistry, Ministry of Health, Saudi Arabia \\ ${ }^{3}$ Specialist in Pediatric Dentistry, General Directorate of Dentistry, Ministry of Health, Saudi Arabia
}

*Corresponding author: Dr. Mohammed S Aldossary, General Dentist, General Directorate of Dentistry, Ministry of Health, Riyadh, Saudi Arabia; Specialist in Pediatric Dentistry, General Directorate of Dentistry, Ministry of Health, Saudi Arabia

\begin{abstract}
Rapid palatal expanders are a widely used orthodontic supplement treatment of posterior cross-bites. Rapid palatal expanders may interfere with sufficient tongue movement and linguo-palatal contact. The aim of this review article was to determine what specific impact rapid palatal expanders have on speech sound production and development in children and early adolescents who had such an orthodontic intervention. Only a few studies have investigated this issue in the literature, generally showing a negative impact on some speech sounds in the very short-term, within two weeks after the placement of the expanders. There is still a shortage in the scientific knowledge regarding the long-term impact and the effect on a wider range of speech sound categories, necessitating further research.
\end{abstract}

\section{Keywords}

Phonetics, Rapid maxillary expansion, Speech acoustic, Voice quality

\section{Introduction}

In orthodontics, it is important to properly inform patients about what to expect and be aware of any potential adversities emerging from using orthodontic appliances. The complications of speech arising from the use of rapid palatal expanders are generally overlooked. One example of rapid palatal expanders is the Hyrax, which is banded over the palate to the bilateral maxillary first molars, connecting to a central jackscrew mechanism and used to expand the narrow maxillary arch [1]. This relatively bulky appliance may interfere with the proper tongue to palate contact required for normal speech sound production.

This article will briefly review the impact that rapid palatal expanders have on speech sound production and development in children and early adolescents who had such an orthodontic intervention. The introductory report includes a brief illustration about sound categories (consonants and vowels), sound analysis, and overview of rapid palatal expanders before reviewing the impact of palatal expanders on speech sound production.

\section{Sound categories and speech analysis}

Speech production is controlled and regulated by over 100 muscles distributed through the chest, abdomen, head and neck. The creation of speech production involves three main functional systems: Respiratory, laryngeal, and supra-laryngeal. Spoken word in its original form is generated by air pressure variations [2]. Air flows from the larynx to the supra-laryngeal system and is acted on by one of the moving structures called articulators [3].

Sounds in American English could be grouped into two categories: Consonants and vowels. Consonants are differentiated from vowels based on the extent to which the articulators cause airway constriction. That results in much more consonant articulation defects than vowels [4]. The consonant articulation is categorized into three basic dimensions, according to Bloodstein: Place, manner, and voice [4]. 
Table 1: Examples of categorization of American English consonant and vowel sounds.

\section{Consonant sounds}

\begin{tabular}{|c|c|c|c|c|c|}
\hline \multirow{2}{*}{$\begin{array}{l}\text { Manner of } \\
\text { articulation }\end{array}$} & \multicolumn{5}{|c|}{ Position of articulation } \\
\hline & Bilabial & Labiodental & Lingua-alveolar & Linguopalatal & Linguavelar \\
\hline Stop-plosives & /p/-pen & - & /t/-top & - & $/ \mathrm{k} /$-çall \\
\hline Fricatives & - & /f/-fill & /s/-sun & IJ/-she & - \\
\hline \multicolumn{6}{|l|}{ Vowel sounds } \\
\hline \multirow[t]{2}{*}{ Tongue height } & \multicolumn{5}{|c|}{ Tongue advancement } \\
\hline & \multicolumn{2}{|l|}{ Front } & \multicolumn{3}{|l|}{ Back } \\
\hline High & \multicolumn{2}{|l|}{ /i/-eat } & \multicolumn{3}{|l|}{ /u/-suit } \\
\hline Mid & \multicolumn{2}{|l|}{ /e/-vacation } & \multicolumn{3}{|l|}{ /o/-obey } \\
\hline Low & \multicolumn{2}{|l|}{ /æ/-ât } & \multicolumn{3}{|l|}{ /a/-father } \\
\hline
\end{tabular}

A vowel is defined as "a voiced sound in forming which the air issues in a continuous stream through the pharynx and mouth, there being no obstruction and no narrowing such as would cause audible friction" [5]. Vowels are produced by vocal tones, which are altered by changes in tongue position in the oral cavity [6]. It is vital to know that the range of formation of certain vowel phonemes is individually variable. Pending different speakers and dialectal variations, the spectrum of these vowels blends by fine degrees, and overlaps can be detected [4]. Due to the unique vocal tract form and changing tongue posture, the phonetic differences are observed [7]. While the position of the tongue mainly distinguishes vowels, the configuration of the lip will alter the acoustics of the vowel sounds [8]. Examples of categorization of American English consonant and vowel sounds are presented in (Table 1).

While voiceless fricatives are produced by altering a turbulent source of noise, vowels have distinct bands of acoustic frequencies called formants. Usually, the center frequencies of these formants are used to discreetly acoustically describe a vowel [9]. Three popular formants of frequency are considered the most relevant to recognize. The lowest frequency band, F1, is related to the height of the tongue when producing a particular vowel. When graphed in a standard F1/F2 plot, decreasing F1 values (moving upward along the $y$-axis) correspond to increases in tongue height. $\mathrm{F} 2$ is related to the advancement of anterior posterior tongue. Increasing $\mathrm{F} 2$ values on the $\mathrm{X}$-axis leads to more anterior locations of the constriction of the primary tongue to the palate. In rhotic sounds, F3 values are significant. Due to differences in anatomical vocal tract size, the exact formant frequencies differ across speakers. Though for a specific vowel, the relative positions of formant frequencies are usually retained among speakers, which allows for analysis [9].

To observe the continuously changing acoustic elements of speech, an electronic recording may be made with the use of a voice spectrogram [7]. Acoustic analysis is an important method used for quantitatively defining discordant speech output [8]. Spectral moment analy- sis is useful to measure and differentiate between voice signals. The spectral moment analysis has been shown to be useful in making an objective distinction between the stop-plosive and fricative consonant groups [10]. Of two reasons, the use of spectral moment analysis of fricative consonants is especially useful: It offers objective data that can be used to demonstrate clinically important changes in voice, and it can differentiate between consonant sounds that may be imperceptible to the human ear [11].

Sound spectrograms (sonograms) are 3D patterns that visually represent time (horizontal axis), frequency or pitch perception (vertical axis), and intensity shown as degree of blackness [8]. Measures of vocal tract resonance (formant center frequencies) are obtained for vowels, and measures of noise distribution (spectral moments) are obtained for consonants using spectrographic analysis software, such as TF32-Computer Program [12].

\section{Overview of rapid palatal expanders}

A rapid palatal expander is typically used in young, growing patients with a posterior cross-bite [13]. Palatal expansion is now being advocated to correct posterior cross-bites to redirect the development of teeth into natural occlusion, remove any functional problems upon contact, trigger beneficial dento-skeletal improvements in a growing patient to minimize potential complexity of treatment [14], and minimize the likelihood of skeletal mandibular asymmetry [15].

With respect to the appliance design, there are three main types of fixed palatal expanders containing jackscrews: The Haas, Hyrax, and bonded types. Both Hass and Hyrax palatal expanders are supported by bands cemented around the maxillary first molars or even the first premolars [16]. The bonded palatal expander does not require bands and is fixed with cement over the occlusal surfaces of the posterior teeth. Regardless of the type of palatal expander, a central jackscrew stretches across the palate and is soldered to the bands, which is activated at home by patients or their parents. The Haas expander is similar to the Hyrax design but also covers 
the palate with acrylic [17]. The Hyrax expander is more hygienic than the Haas since it is an all wire frame and induces the least irritation to the palatal mucosa [13]. Certain expanders come as a removable plate with a central jackscrew or heavy spring, or a lingual arch, such as a quad helix or W-arch [18].

When considering a palatal expander, the timing for treatment intervention is critical because growth ceases at different times. During the younger years, palatal expansion is more vital because the transverse dimension is the first to cease. Any expansion intervention will have a high success before pubertal growth, separating the mid-palatal suture; however, a heavier force applied from a jackscrew is more successful during adolescence [18]. The optimal upper age for expansion is $13-15$ years of age; however, expansion in older patients may be possible but at a less predictable level [13].

\section{Impact of rapid palatal expanders on speech sound production}

Before discussing the impact of palatal expanders on sound production, it is interesting to briefly explore the impact of palatal shape on speech. A study by Lubit [19], examined individuals with high or narrow palates and concluded that they more commonly have speech disorders. Another study by Laine [20], concluded that the $/ \mathrm{s} /$ sound in subjects with a narrower palate in all segments was distorted at a higher rate. One explanation for this distortion is less available space for the tongue movements required for proper speech. Laine also found that medio-alveolar consonants are somewhat affected by the size of the maxillary arch but not the mandibular arch [20]. Furthermore, there was no significant association between speech errors and malocclusion, molar classification, overjet, overbite, anterior cross-bite, spacing, or crowding in a recent study in a sample of 115 untreated individuals [21].

One of the main drawbacks of a palatal expander that includes a jackscrew is its bulkiness in the palatal region [18]. Since nearly $90 \%$ of all consonants are articulated in the anterior part of the oral cavity, this can lead to temporary speech difficulties [21]. This is particularly important as there is less flexibility to produce consonant sounds as opposed to vowels, as consonants require a more precise positioning of the tongue [22]. Such information can be shared with the patient undergoing orthodontic treatment during initial pre-treatment counseling and help with interdisciplinary counseling for orthodontic patients receiving palatal expanders. Previous research using an intraoral bite block and artificial palate have shown that the production of consonants is more affected than vowels $[22,23]$.

To date, only very few studies have explored the speech production and its relationship with rapid palatal expanders. One research by De Felippe, et al. [24] included a retrospective questionnaire given to $165 \mathrm{pa}$ - tients receiving various designs of fixed rapid palatal expanders. From a patient's perspective, nearly $89 \%$ of patients claimed that their speech was initially affected by the expander during the first week after cementation, regardless of the type of expander. However, it was difficult to decide what phonetic sounds and to what extent the speech was impaired. Following one week, the majority of patients self-reported their speech returned to normal, indicating a remarkable adaptation to the appliance. Additionally, age and gender factors did not influence the variables [24].

Another more clinically based study by Stevens, et al. [25] identified the speech alterations in 22 patients who received Hyrax or bonded type expanders. Speech recordings were taken at 6 time points: before placement of the expander, after insertion, during expansion, during retention, after removal, and 4 weeks following removal. Speech acceptability was assessed by 10 native undergraduate students as well as an acoustic analysis conducted for fricatives $/ \mathrm{s} /, / \mathrm{s} /$, and $/ \mathrm{i} /$ vowel sounds. In general, once the appliance was first placed, speech was altered and distorted. The patients' speech gradually improved over time and returned to baseline once the appliance had been removed. These three sounds were considered to be affected to some degree in the production of speech sound. The affected vowel, /i/, showed a decrease for $F 2$ from Time 1 (before expander placement; T1) to Time 2 (immediately after cementation; T2), and also had a significant increase in F1 from T1-T2 [25]. Moreover, they analyzed spectral moments for $/ \mathrm{s} /$ and $/ \mathrm{s} /$ and found significant distortions. It wasn't until 2-4 weeks post insertion that adaptation occurred at levels similar to baseline. Although patients learned to adjust tongue positions for some phonemes, those phonemes did not fully return to baseline [25].

With specific phonemes, interdisciplinary collaboration with a speech pathologist may be more focused on helping with tongue location changes. It was noted that patients do not learn to change the tongue position within a 2-week span for the palatal expansion of all sounds.

Assuming that tongue height is the primary factor associated with the expander interference, we might expect to see consonants and high voiced vowels being the most affected, followed by mid-high vowels and finally, low-mid vowels being the least affected during the placement of a rapid palatal expander.

The previous two studies [24,25], have utilized perceptual analysis to rank the degree of speaker impairment on a scale, which has limitations regarding accuracy and reliability $[26,27]$. Anacoustic approach to data analysis allowed for a more objective appraisal of the phoneme-specific effects of the palatal expander.

More recently, in a cross-sectional study by Macari, et al. [28], the sounds of a total of 14 patients (aged 9.6 
to 15 years) with constricted maxilla undergoing rapid palatal expansion were investigated. They found a significant lowering (decreased) of the first and second formants (F1 and F2) for the vowel /a/ in $78.6 \%$ and $71.4 \%$ of participants, respectively [28].

In another study conducted by Biondi, et al. [29] the authors examined possible changes in phonetics induced by rapid palatal expanders. Thirty-five patients (aged 7-14 years, mean $9.3 \pm 2.3$ years), scheduled for rapid palatal expansion, were divided into two groups: Banded two-arm Hyrax and banded four-arm Hyrax. Speech samples were obtained before insertion, during treatment and after expander removal, up to 6 months post fitting, and 2 months after expander removal. Acoustical analysis was carried out using PRAAT and BioVoice analysis tools. They found that rapid palatal expansion causes a slight phonetic change in the acoustical parameters of both consonants and vowels. During therapy, the two-arm Hyrax caused less speech impairment than the four-arm Hyrax [29].

Interestingly, on the other hand, Yurttadur, et al. [30] in their controlled clinical study, found no statistically significant differences between the treatment and control groups among all voice parameters. In a total of 40 patients ( 20 for treatment group, 20 for control group), they prospectively assessed the effect of rapid palatal expansion on vocal function. Acoustic voice samples were recorded from all patients at $\mathrm{T} 1$ and $\mathrm{T} 2$ by the Multi-Dimensional Voice Program for acoustic analysis in a Computerized Speech Lab. They concluded, therefore, that rapid palatal expansion does not affect vocal quality or resonance, so it can be safely used with patients [30].

\section{Recommendations for future research}

Based on the limitation of the available studies in the literature, we recommend the following for future studies. 1) Looking at a wider variety of consonants as well as a more comprehensive list of vowel sounds to better understand the impact of rapid palatal expanders on the production of speech sounds; 2) Rather than relying on patients' perspectives, we recommend using digital devices for speech recordings and analyses, such as using a phonetically balanced reading passage, "The Caterpillar" [31], and Sound spectrograms [8,12]; 3) A longitudinal trial to monitor patients for a longer period of time to investigate the long-term effect or reliably define the duration of the temporary alteration in sound production, i.e., when will the affected phonemes returned to baseline?; 4) Investigating a larger sample size to look across more potential influencing factors, such as age, gender, and native language.

\section{Conclusions}

From the very limited number of studies in the literature regarding the impact of palatal expanders on sound production, we can conclude that numerous phonemes can be distorted upon the expander's placement, which indicated altered speech sound production. For most phonemes, it takes longer than two weeks for speech to return to baseline, if at all. Future research may incorporate the inclusion of more phonemes, a longer study length, and a larger sample size to look across more potential influencing factors.

\section{Ethics Approval}

Not Applicable.

\section{Funding}

None.

\section{Conflicts of Interest}

The authors have no actual or potential conflicts of interest.

\section{Authors Contribution}

Authors have contributed equally to this review article.

\section{References}

1. Agostino P, Ugolini A, Signori A, Silvestrini-Biavati A, Harrison JE, et al. (2014) Orthodontic treatment for posterior crossbites. Cochrane Database Syst Rev.

2. Vance M, Stackhouse J, Wells B (2005) Speech-production skills in children aged 3-7 years. Int J Lang Commun Disord 40: 29-48.

3. DeVeney SL (2019) Clinical challenges: Assessing toddler speech sound productions. Semin Speech Lang 40: 81-93.

4. Bloodstein O (1984) Speech pathology an introduction. ( $2^{\text {nd }}$ edn), Houghton Mifflin Company.

5. Roach P (2004) Phonetics. Oxford University Press, 73-74.

6. Travis LE (1971) Handbook of speech pathology and audiology. Prentice-Hall Englewood Cliffs, NJ.

7. Skinner PH, Ralph LS (1985) Speech, language, and hearing: Normal processes and disorders. John Wiley \& Sons.

8. Shriberg LD, Kent RD, Munson B (2003) Clinical phonetics. Allyn and Bacon Boston.

9. Neel AT (2010) Using acoustic phonetics in clinical practice. Perspect Speech Sci Orofac Disord 20: 14-24.

10. Forrest K, Weismer G, Milenkovic P, Dougall RN (1988) Statistical analysis of word-initial voiceless obstruents: Preliminary data. J Acoust Soc Am 84: 115-123.

11. Mandulak KC (2011) "I can see what you're saying": Clinical utility of spectral moment analysis. Perspect Speech Sci Orofac Disord 21: 44-54.

12. Milenkovic P (2004) TF32. Madison, WI Univ Wisconsin--Madison.

13. Bishara SE, Staley RN (1987) Maxillary expansion: Clinical implications. Am J Orthod Dentofacial Orthop 91: 3-14.

14. Bell RA (1982) A review of maxillary expansion in relation to rate of expansion and patient's age. Am J Orthod 81: 32-37.

15. Kilic N, Kiki A, Oktay H (2008) Condylar asymmetry in unilateral posterior crossbite patients. Am J Orthod Dentofac Orthop 133: 382-387. 
16. Ngan PW, Wei SH (1990) Treatment of posterior crossbite in the primary and early mixed dentitions. Quintessence Int 21: $451-459$.

17. Haas AJ (1970) Palatal expansion: Just the beginning of dentofacial orthopedics. Am J Orthod 57: 219-255.

18. Proffit WR, Fields HW, Larson B, Sarver DM (2018) Contemporary orthodontics-e-book. Elsevier Health Sciences.

19. Lubit EC (1967) The relationship of malocclusion and faulty speech articulation. J Oral Med 22: 47-55.

20. Laine $T$ (1986) Articulatory disorders in speech as related to size of the alveolar arches. Eur J Orthod 8: 192-197.

21. Leavy KM, Cisneros GJ, LeBlanc EM (2016) Malocclusion and its relationship to speech sound production: Redefining the effect of malocclusal traits on sound production. Am J Orthod Dentofacial Orthop 150: 116-123.

22. McFarland DH, Baum SR (1995) Incomplete compensation to articulatory perturbation. J Acoust Soc Am 97: 18651873.

23. Baum SR, McFarland DH (2000) Individual differences in speech adaptation to an artificial palate. J Acoust Soc Am 107: 3572-3575.

24. De Felippe NLO, Da Silveira AC, Viana G, Smith B (2010) Influence of palatal expanders on oral comfort, speech, and mastication. Am J Orthod Dentofacial Orthop 137: 48-53.
25. Stevens K, Bressmann T, Gong SG, Tompson BD (2011) Impact of a rapid palatal expander on speech articulation. Am J Orthod Dentofacial Orthop 140: 67-75.

26. Kent RD (1996) Hearing and believing: Some limits to the auditory-perceptual assessment of speech and voice disorders. Am J Speech-Language Pathol 5: 7-23.

27. Kreiman J, Gerratt BR, Precoda K (1990) Listener experience and perception of voice quality. J Speech Hear Res 33: $103-115$.

28. Macari AT, Ziade G, Khandakji M, Tamim H, Hamdan AL (2016) Effect of rapid maxillary expansion on voice. J Voice 30: 760 .

29. Biondi E, Bandini A, Lombardo L, Orlandi S, Siciliani G, et al. (2017) Phonetic analysis during treatment with rapid maxillary expander. Orthod Craniofac Res 20: 21-29.

30. Yurttadur G, Basciftcı FA, Ozturk K (2017) The effects of rapid maxillary expansion on voice function. Angle Orthod 87: 49-55.

31. Rupal Patel, Kathryn Connaghan, Diana Franco, Erika Edsall, Dory Forgit, et al. (2013) "The caterpillar": A novel reading passage for assessment of motor speech disorders. Am J Speech-Language Pathol 22: 1-9. 